\title{
Intersections
}

Canadian Journal of Music

Revue canadienne de musique

\section{Vincent d'Indy et son temps. 2006. Dirigé par Manuela \\ Schwartz. Collection « Musique-Musicologie ». Liège : Mardaga. \\ 391 p. ISBN 2-87009-888-X (couverture souple)}

\section{Jon-Tomas Godin}

Volume 27, numéro 2, 2007

URI : https://id.erudit.org/iderudit/1013116ar

DOI : https://doi.org/10.7202/1013116ar

Aller au sommaire du numéro

Éditeur(s)

Canadian University Music Society / Société de musique des universités canadiennes

ISSN

1911-0146 (imprimé)

1918-512X (numérique)

Découvrir la revue

Citer ce compte rendu

Godin, J.-T. (2007). Compte rendu de [Vincent d'Indy et son temps. 2006. Dirigé par Manuela Schwartz. Collection " Musique-Musicologie ». Liège : Mardaga. 391 p. ISBN 2-87009-888-X (couverture souple)]. Intersections, 27(2), 88-92.

https://doi.org/10.7202/1013116ar

Copyright (C Canadian University Music Society / Société de musique des universités canadiennes, 2008
Ce document est protégé par la loi sur le droit d'auteur. L'utilisation des services d'Érudit (y compris la reproduction) est assujettie à sa politique d'utilisation que vous pouvez consulter en ligne.

https://apropos.erudit.org/fr/usagers/politique-dutilisation/ 
Vincent d'Indy et son temps. 2006. Dirigé par Manuela Schwartz. Collection " Musique-Musicologie ». Liège : Mardaga. 391p. ISBN 2-87009-888-X (couverture souple).

Depuis l'affirmation du courant de la New Musicology ${ }^{1}$, les chercheurs nordaméricains s'intéressent davantage aux compositeurs écartés par le canon consacré par la tradition classique. 'Parmi ceux-ci, Vincent d'Indy occupe une place croissante. D'Indy n'est bien sûr pas inconnu des musicologues, son activité pédagogique et administrative au sein d'institutions aussi importantes que la Schola Cantorum ou la Société Nationale ayant fait l'objet d'études plus ou moins détaillées ${ }^{2}$. C'est plutôt d'Indy compositeur, relégué dans l'oubli depuis sa mort, qui est maintenant réhabilité.

Létude de d'Indy pose plusieurs problèmes sur lesquels se sont penchés les participants du colloque Vincent d'Indy et son temps, tenu à la Bibliothèque Nationale de France en 2002. Mardaga en publie ici les actes. Comme le souligne Manuela Schwartz dans son introduction (p. 7-17), d'Indy possède une personnalité complexe qui, malgré ses polémiques clairement rédigées, demeure ambiguë et contradictoire. Que ce soit l'incompatibilité apparente entre ses positions antisémites et son amitié avec des Juifs comme Paul Dukas ou la famille Cahen, ou encore entre son profond catholicisme et le petit nombre d'œuvres sacrées qui figurent à son catalogue, les paradoxes abondent. Cet ouvrage n'a cependant pas pour objectif de les expliquer, mais vise plutôt à jeter un nouvel éclairage sur plusieurs aspects de la vie et de l’œuvre de cet artiste encore méconnu à l'extérieur de la France.

Lédition d'actes de colloque en volume pose souvent des défis d'unité et de cohérence auxquels Schwartz a trouvé des solutions élégantes. Outre la grande diversité des textes, la tentation de les regrouper d'après les sessions du colloque donne souvent de pauvres résultats. En raison des propos parfois contradictoires qui peuvent s'insérer dans les communications d'un colloque, la simple transcription des textes proposés peut souffrir d'un manque de continuité. Un effort doit être fait pour agencer les textes de façon à dégager une logique dans la suite des chapitres ou des entrées, logique presque impossible à atteindre dans le cadre d'un colloque. Cependant la division de louvrage en cinq sections, quelle reprenne ou non les sessions du colloque, maintient ici une parfaite cohérence.

Outre la question de l'hétérogénéité et de l'agencement des textes, celle de la sélection peut aussi poser problème. Dans un souci d'offrir une image aussi complète que possible du colloque et du compositeur qui en fait l'objet, ce recueil contient toutes les communications présentées au colloque, sauf trois (parmi ces dernières, la contribution de Jann Pasler a depuis été publiée ailleurs ${ }^{3}$ ). Les

1 Voir à ce propos le bilan que dresse Jean-Jacques Nattiez dans son article « Histoire ou histoires de la musique? " (2006, 19-49).

2 La bibliographie qui accompagne ce compte rendu propose une liste de références sur ce sujet.

3 La matière de sa communication a fait lobjet de deux articles depuis 2005, "Déconstruire d'Indy " et " Deconstructing d'Indy, or the Problem of a Composer's Reputation ", publiés dans la Revue de musicologie et 19th-Century Music (voir Pasler 2005 et 2007). A ma connaissance, les deux autres conférences (de Renate Groth et de Florence Le Doussal) demeurent inédites. 
contributions retenues prennent des formes variées : études approfondies des réseaux de contacts du compositeur, analyses des œuvres ou encore description des voyages entrepris ou de la discographie.

J'aborderai le contenu de Vincent d'Indy et son temps en me penchant sur trois volets : les réseaux sociaux de d'Indy, sa relation avec la musique religieuse et les compositeurs qui lui ont servi de modèles.

\section{L'HOMME SOCIAL}

Parmi les étiquettes qu'on accole le plus fréquemment à d'Indy, celle du conservateur réactionnaire mérite dêtre réexaminée. L'influence de la situation familiale - aristocrate et aisée, avec une longue tradition militaire - compte certainement pour quelque chose, et l'article de Myriam Chimènes témoigne de cette ascendance du compositeur sur le milieu parisien (p. 65-85). Chimènes montre aussi que d'Indy fréquentait un nombre important de salons de la capitale. Ces salons, bourgeois ou aristocratiques, constituaient un lieu de diffusion de ses œuvres et de ses idées ${ }^{4}$, ainsi qu'une source d'appui indispensable pour concrétiser ses projets. L'image conservatrice de d'Indy est ici resituée dans son contexte et une distinction plutôt claire entre la polémique entourant d'Indy et ses affiliations sociales en émane. Loin dêtre conservateur, d'Indy fréquentait plusieurs salons (ceux de la comtesse de Chambrun, de Marguerite de Saint-Marceaux ou de la cantatrice Jeanne Max parmi plusieurs) et semblait à l'aise dans des milieux sociaux et politiques très variés.

La situation familiale de d'Indy n'influença pas que son environnement parisien. Comme le démontre Esteban Buch (p. 21-36), le compositeur arborait une conception particulièrement romantique de la guerre provenant au moins en partie du milieu privilégié auquel il appartenait. Cette conception contraste étonnamment avec la dure réalité des tranchées de la Première Guerre mondiale. L'exemple fascinant de la composition de la Sinfonia Brevis de bello gallico, plus particulièrement des impressions héroïques que retient d'Indy après une visite au front et de leur rôle dans la genèse de l’œuvre, indique la puissance de l'imagination du compositeur, mais aussi la fragilité du lien qui unit le programme écrit et l'œuvre. Buch retrace avec soin les étapes de l'évolution de l'œuvre ainsi que ses premières exécutions, et explique combien il était difficile pour l'auditoire de déceler l'intention de l'auteur sans avoir lu le programme. Ce qui avait été conçu par d'Indy comme symphonie à programme, avec titre et texte à lappui, devenait une œuvre de musique "pure " lorsqu'on écartait le programme du contexte d'interprétation. Loin de nier la puissance évocatrice de la musique ou le talent du compositeur, cette incompréhension illustre une rupture de communication entre lémetteur et le récepteur qui s'explique facilement par le modèle bien connu de la tripartition ${ }^{5}$. Sans référents explicites, la musique

4 Pour une étude des réseaux d'avant-garde dans lesquels d'Indy a évolué durant l'époque de la Société Nationale de musique, voir Duchesneau 1997.

5 Une explication plus complète du modèle de la tripartition proposé par Molino-Nattiez est développée dans Nattiez 1987. 
demeure un moyen de communication à la fois moins précis et plus évocateur que la parole, idée romantique que d'Indy aurait sans doute appuyée.

Parmi les innovations proposées dans les études d'indystes, cet ouvrage porte une attention toute particulière au réseau de contacts belges du compositeur. Michel Stockhem consacre une étude approfondie aux échanges entre d'Indy et quelques membres influents de la communauté belge autre que son ancien mầtre, César Franck (p. 87-98). Entre autres, les noms de Maurice Kufferath, Octave Maus et Albert Cahen d'Anvers sont cités à plusieurs reprises. La Belgique, avec son public averti et réceptif, son niveau de compétition plus accueillant quà Paris, et ses nombreux mélomanes et promoteurs, offrait au compositeur un lieu idéal pour la diffusion de ses oeuvres. Sans minimiser l'importance de la filière belge, une plus grande place aurait pu être accordée à l'analyse des réseaux dans d'autres pays, aux États-Unis entre autres. James Ross en donne tout de même un aperçu dans son article sur les activités d'interprétation de d'Indy (p. 331), mais une réflexion approfondie sur les voyages américains du compositeur reste à proposer.

\section{LA RELIGION}

Le spectre du catholicisme de d'Indy, une autre image persistante qui hante les spécialistes du compositeur, n'est pas à remettre en question, mais plutôt à resituer dans son contexte culturel. Stéphane Giocanti se pose la question "Vincent d'Indy est-il un compositeur religieux ? " et arrive à des conclusions intéressantes (p. 255-262). D'Indy envisageait de façon très particulière la musique religieuse, ce qui explique en partie une des contradictions apparentes de son corpus, soit l'absence remarquable d'œuvres religieuses. Dans son article, Renata Suchowiejko aborde le concept de la spiritualité dans l'œuvre de d'Indy (p. 101-110), citant en exemple plusieurs passages du Cours de composition musicale qui rendent explicite l'idée d'un art au service de la religion. Pour Suchowiejko, cette vision découle de la notion romantique de la religion de l'art. Quoique d'Indy fut sans doute influencé par cette notion, il demeure ancré dans une tradition beaucoup plus catholique. Pour le compositeur, il devait émaner de la musique un sens de la charité et de l'amour chrétiens, comme par exemple dans L'Étranger, ce que démontre l'article de Steven Huebner (p. 263-282). Le concept de la religion de l'art, au contraire, évolue dans une direction opposée : la représentation du sublime, qui signifiait au départ Dieu et facilitait la contemplation des mystères de la foi, est transformée en représentation de la condition humaine, c'est-à-dire que l'art remplace Dieu comme objet de contemplation.

En lien avec la religion, la question de l'antisémitisme doit être ici abordée. Larticle approfondi de Schwartz retrace les sources de l'antisémitisme et en dresse la typologie (p. 37-64). L'antisémitisme personnel de d'Indy, sans doute le produit de plusieurs influences, est étudié dans ce volume sous l'optique des amitiés de d'Indy avec plusieurs Juifs importants, dont Albert Cahen d'Anvers et Paul Dukas. Ces amitiés permettent encore une fois de distinguer les polémiques publiques et la vie privée du compositeur. Schwartz conclut pour sa part que la question de l'antisémitisme de d'Indy n'est pas épuisée et qu'une étude ap- 
profondie des écrits du compositeur durant la période 1890-1910, ainsi qu'une comparaison des positions de Wagner et de d'Indy restent à entreprendre avant de pouvoir mieux cerner la question.

\section{LES MODÈLES}

Sans réduire le rôle de Franck et Wagner comme modèles chez d'Indy, louvrage s'attarde à des sources d'influences moins connues. C'est ainsi qu'Annegret Fauser retrace les origines de l'historicisme de d'Indy et son incidence majeure sur la structure du Cours de composition musicale (p. 123-33). En développant son concept d'archéologie musicale, Fauser montre comment le travail de réhabilitation d'œuvres anciennes permet à d'Indy d'élaborer une conception cyclique de l'histoire qui traverse le Cours. Cette conception habilite une vision atemporelle de l'art ouvrant la voie à plusieurs rapprochements et au regroupement d'œuvres qui ne pourraient lềtre dans un récit à temporalité plus linéaire. La linéarité nesst cependant pas mise de côté dans la pensée de d'Indy; elle revient sous forme de successions des compositeurs, c'est-à-dire une suite de téléologies menant à quelques grands représentants de chacune des formes - Wagner pour la musique de scène, Franck pour la musique instrumentale. C'est ce que Katharine Ellis explore dans son excellent texte (p. 111-122), notamment la téléologie particulière créée par d'Indy qui retrace l'histoire de la musique dramatique depuis ses débuts jusquà son point culminant, que le compositeur perçoit dans l’œuvre de Wagner.

Outre les thèmes religieux, la nature constitue une autre source d'inspiration dans l'œuvre de d'Indy. Lien avéré au romantisme ${ }^{6}$, la présence de thèmes naturels dans la production du compositeur est mise en relation avec la thématique religieuse dans le Jour deeté à la montagne, puis comparée à Eine Alpensinfonie de Strauss par Stefan Keym. Il en résulte une analyse étonnante qui révèle de multiples influences et une nouvelle compréhension d'un des bijoux du catalogue de d'Indy.

\section{APRÈS D'INDY...}

Le penseur du Cours de composition musicale a laissé à l'histoire bien plus qu'un manuel d'histoire et de composition, qui a marqué plus d'une génération de compositeurs et de chercheurs. L'ouvre musicale de d'Indy, vite tombée dans loubli à cause, entre autres, de l'avènement d'un modernisme plus combatif et des lourdes polémiques suscitées par le compositeur - quon pense à son autoritarisme à travers la Schola ou à la question de l'antisémitisme évoquée plus tôt - mérite une attention qui commence à naitre. Faute d'espace, je n'ai pu discuter les articles d'analyse de Lucile Thoyer (" La musique de chambre de la période d'Agay et le néo-classicisme ", p. 151-70), de Herbert Schneider ("La musique de chambre avec piano dans son contexte historique ", p. 171-93), de Hervé Lacombe ("D'Indy et la musique de scène », p. 211-30) ou de Damien Ehrhardt ("La musique à programme chez Vincent d'Indy », p. 231-42), qui forment la section "Création, formes et genres ", ni celui de Steven Huebner

6 Voir à ce sujet la monographie de Rosen 2002. 
( « Le Hollandais fantôme : Ideology and Dramaturgy in L’Etranger », p. 263-81), qui se trouve dans la section "Sources d'inspiration ». Chacun de ces textes mérite une lecture attentive pour la lumière qu'il jette sur une œuvre ou un genre pratiqué par d'Indy et pour l'évaluation nuancée qu'il propose de compositions souvent méconnues.

Plusieurs articles de ce volume contribuent considérablement à l'avancement de nos connaissances sur d'Indy. Dans la foulée des ouvrages de la mouvance de la New Musicology, ce volume peut servir en quelques points de modele aux études dédiées à des compositeurs non canoniques. En tant qu'ouvrage collectif, il permet une diversité d'approches qui enrichit notre appréciation du compositeur. Lattention portée aux multiples facettes de la personnalité et de la production de d'Indy, très appropriée chez un personnage aussi prolifique, demeure essentielle à la connaissance de tout artiste. La pluralité des informations présentées ainsi que la qualité des réflexions font de Vincent d'Indy et son temps un ajout intéressant à la bibliothèque d'un chercheur. La réévaluation du compositeur amorcée dans ce volume ouvre de nouvelles pistes qui permettront sans doute à plusieurs chercheurs de mieux faire connaittre ce maître oublié.

\section{RÉFÉRENCES}

Biron, Fernand. 1941. Le chant grégorien dans l'enseignement et les œuvres musicales de Vincent d'Indy. Ottawa : Éditions de l'Université d'Ottawa.

Demuth, Norman. 1974. Vincent d'Indy 1851-1931 : Champion of Classicism. $2^{\mathrm{e}}$ éd. Westport, CT : Greenwood Press.

Duchesneau, Michel. 1997. L'avant-garde musicale et ses sociétés d Paris de 1871 d 1939. Hayen : Mardaga.

Indy, Vincent d', Auguste Sérieyx et Guy de Lioncourt. 1912-1950. Cours de composition musicale. 4 vol. Paris : Durand.

Maillard, Jean et Francine. 1994. Vincent d'Indy : le Maître et sa musique, la Schola Cantorum. Bourg-la-Reine, Éditions Auguste Zurfluh.

Nattiez, Jean-Jacques. 1987. Musicologie générale et sémiologie. Paris : Christian Bourgois.

_. 2006. "Histoire ou histoires de la musique ? ". Dans Musiques. Une encyclopédie pour le XXIe siècle, "4. Histoire des musiques européennes", sous la dir. de Jean-Jacques Nattiez, 19-49. Arles-Paris : Actes Sud/Cité de la musique.

Pasler, Jann. 2005. "Déconstruire d'Indy ". Revue de musicologie 91/2 : 369-400.

- 2007. "Deconstructing d'Indy, or the Problem of a Composer's Reputation ». Nineteenth-Century Music 30/3 : 230-56.

Pommiès, Élisabeth. 2001. Vincent d'Indy. Paris : Séguier.

Rosen, Charles. 2002. La génération romantique. Paris : Gallimard.

Thomson, Andrew. 1996. Vincent d'Indy and His World. Oxford : Clarendon Press.

Vallas, Léon. 1950. Vincent d'Indy. 2 vol. Paris : Albin Michel. 